\title{
ESTUDIO SOBRE LOS PROCESOS DE INTEGRACIÓN LABORAL DE LOS INMIGRANTES
}

\author{
STUDY OF IMMIGRANTS' LABOUR INTEGRATION PROCESS
}

\author{
Assumpta Aneas* y Trinidad Donoso** \\ Universidad de Barcelona
}

\begin{abstract}
RESUMEN
El presente trabajo pretende mostrar los resultados de un estudio cuya intención era identificar posibles variables que afectan a los procesos y estrategias de integración laboral de personas procedentes de otros países; las necesidades formativas en relación al colectivo de referencia y ampliar el conocimiento del fenómeno en aras de la intervención psicoeducativa y social. A partir de una metodología de investigación de orientación cualitativa se ha concluido que las diferencias en cuanto a las estrategias de integración y sus logros están más determinadas por los niveles de cualificación en el país de origen que por las propias diferencias culturales.
\end{abstract}

Palabras clave: inmigración, trabajador inmigrante, Integración laboral, investigación cualitativa.

\begin{abstract}
The article shows the results of a study carried out by the authors to identify possible variables that affect the processes and strategies of occupational integration of people coming from other countries, to establish training needs in relation to the reference group and to extend the knowledge of the phenomenon for the sake of the educational and social intervention.

Using a qualitative research methodology, it was concluded that the differences in integration strategies and their accomplishments are better explained by the levels of qualification attained in the country of origin than by cultural differences.
\end{abstract}

Key words: immigration, migrant worker, occupational integration, qualitative research.

* Assumpta Aneas es profesora lectora de la Facultad de Pedagogía de la Universidad de Barcelona, posee un largo historial profesional en la formación ocupacional y continua, así como la inserción laboral. Colaborando y dirigiendo proyectos para colectivos de jóvenes, mujeres desempleadas y parados de larga duración. Desarrolló su tesis doctoral estudiando las competencias interculturales en las organizaciones. Actualmente, entre sus líneas de investigación destacan, las competencias profesionales y la interculturalidad en las organizaciones. aaneas@ub.edu

** Trinidad Donoso es profesora Titular de la universidad de Barcelona. Líneas de investigación preferentes en temas afines al diagnóstico y la orientación profesional: construcción y validación de instrumentos, formación e inserción sociolaboral, formación intercultural y competencias interculturales, género y ciudadanía, entre otras. trinydonoso@ub.edu 


\section{Introducción}

La finalidad del presente trabajo es aproximarse a los procesos y estrategias de integración socio-laboral de personas procedentes de otros países.

La población inmigrante encuentra en nuestro país un contexto legal ciertamente complejo. Este marco legal determina contundentemente las posibilidades de regularización y consiguientemente de integración y la emergencia de nuevos retos y necesidades en las empresas multiculturales (Aneas, 2003).

El presente análisis puede permitir establecer necesidades formativas en relación al colectivo de referencia y ampliar nuestro conocimiento de la realidad en aras de la intervención psicopeducativa y social. Se apuntan, a continuación, unas reflexiones sobre el fenómeno de la interculturalidad y la inserción socio-laboral.

\section{Algunos aspectos básicos sobre globalización, interculturalidad e inserción}

\section{Globalización-interculturalidad}

Las economías de todo el mundo se han hecho interdependientes a escala global, introduciendo una nueva forma de relación entre economía, Estado y sociedad en un sistema de geometría variable (Castells, 2000).

Las consecuencia e hitos sociales vinculados a este fenómeno se están manifestando en la acentuación del desarrollo desigual no sólo entre Norte y Sur (Arango, 2002) sino también entre los segmentos más dinámicos de las sociedades (Castells, 2000).

Ello está incrementando exponencialmente el número de refugiados y emigrantes que huyen de la pobreza y la represión (Aja, 1994; Baldwin-Edwards, 2002; Bauböck, 2002; Böcker, 1998; (UNHCR), 2001 y Ahmed, 2002).

Frente a esta situación, la gente tiende a reagruparse en torno a identidades primarias: religiosa, étnica, territorial, nacional, tal y como han recogido Castells (2000); Calhoun (1994); Touraine (1994) y otros.

Las consecuencias de todos estos cambios en el escenario productivo y social de la era post-industrial generan una percepción y una conciencia de complejidad que afectan a la empresa y la sociedad en general (Donoso, Aneas, 2003; Francesco, 1999).

\section{Inserción socio-laboral}

El término Inserción socio-laboral engloba dos procesos, la aceptación y asimilación de los individuos por parte de la comunidad o el medio social, estructurando un sistema que permite tomar parte en la misma con todos los derechos y deberes, e integración laboral que ve a las personas como fuerza activa de trabajo. Por otra parte, el término va ligado al de exclusión (marginación, vulnerabilidad), identificado como el aparcamiento de la vida social, pérdida o ausencia de autonomía e independencia para conseguir los recursos necesarios, integrarse y participar (García y Figuera, 2000).

Al tratar la inserción socio-laboral de la población inmigrante deben barajarse: la estructura de la sociedad concreta en la que se desarrollan sus dinámicas y la actuación de las empresas. 
Sabemos que la vulnerabilidad de los diferentes colectivos a las situaciones críticas y a los factores de riesgo de exclusión está relacionada con la disponibilidad, accesibilidad y adecuación de los recursos a sus necesidades y demandas (Donoso, Figuera, 2005).

\section{Método}

\section{Objetivos del estudio}

Los objetivos a desarrollar en el presente trabajo son:

a) identificar las variables que afectan la integración del trabajador inmigrante en la empresa y

b) conocer las estrategias de localización y acceso a una ocupación en un país que no es el propio.

Dados los objetivos del presente trabajo, se estimó que la metodología cualitativa era la que contribuiría mejor a la consecución de los objetivos descriptivos y comprensivos del mismo.

Como estrategia de recogida de información se ha utilizado una entrevista personal semi-estructurada.

En el diseño de la misma se ha intentado incluir reactivos para obtener información acerca de: la Preparación del proyecto migratorio, los procesos de regularización y las estrategias de inserción.

\section{Muestra}

La muestra la conforman un total de 35 personas, repartidas entre baja, media y alta cualificación. Se ha entrevistado población autóctona (18) y inmigrante (17) para conocer también el punto de vista de los mandos.

Los niveles de la población autóctona que hemos entrevistado son los siguientes: Director RRHH, Gerente, técnico del Servei català de colocació, responsable de formación, responsable de selección, responsable de comité de empresa.

Países de origen de las personas entrevistadas: Ucrania, Marruecos, Colombia, Perú, México, Ecuador, Lituania, Brasil, Argelia, Camerún. La localización de los informantes clave que hemos entrevistado se ha efectuado a partir de las técnicas de "bola de nieve" aplicada a partir de las propias empresas y agentes clave que han colaborado con nosotros con otras fases del proyecto.

\section{Resultados}

\section{Nivel de ocupabilidad}

Según Blanch (1990) puede predecirse la empleabilidad a partir de una serie de variables latentes que conforman el perfil subjetivo individual del sujeto, como son los valores y 
valencias laborales, la disponibilidad al empleo, la autoimagen profesional, las atribuciones empleo-desempleo, y el estilo de afrontamiento de la búsqueda de trabajo.

En nuestro caso cabe señalar que todos los hombres entrevistados, exceptuando Alfredo de Colonia (de 21 años y estudiante en su país de origen) —en el caso de las mujeres no hemos visto esta dimensión tan claramente-, tenían una amplia experiencia laboral en su país de origen o en España (las diferencias se fundamentan sobre todo en la edad de la persona, a mayor edad, más experiencia laboral en el país de origen). La totalidad de las personas a las que hemos entrevistado han trabajado en diversas actividades y ocupaciones (sectores, horarios, etc.) tanto en nuestro país como en el país de origen.

Los trabajadores veían el trabajo como el medio para su subsistencia, y en este sentido los valores instrumentales del trabajo eran los primeros que aparecían, pero exhibían unos valores relacionados con el trabajo bastantes expresivos, se verá también mas adelante, en estrecha consonancia con los resultados de la investigación de Risquez, (2004) con una muestra de trabajadores irlandeses.

La autoimagen profesional de muchos se debatía entre la autoeficacia positiva y la melancolía "Yo era ingeniero mecánico, era el responsable de una fábrica bastante grande, ahora estoy como mozo de mantenimiento" (Igor, Ucrania). "Yo era analista informático. Controlaba el servicio informático de una gran empresa" (Victor P. Perú). "Yo era ingeniera de fluidos" (Odeta, Lituania).

En cuanto a las atribuciones que realizan están influenciadas por esa autoeficacia positiva que les hace verse a sí mismos como personas con "ganas de trabajar, capacidad de trabajo, honradez, honestidad, etc.". Muchos de ellos también han hecho una serie de alusiones, que consideramos interesantes y significativas acerca de la suerte de haber encontrado o conocido, aquí en España, gente buena, amable y honrada que les ha ayudado. Encontramos interesante esta valoración no por ser un juicio positivo hacia la población autóctona. Estos comentarios denotan una percepción positiva y una sensibilidad para identificar de qué persona se puede confiar, y para sobre todos los avatares, recordar lo positivo. Al respecto, Victor B recordaba con cariño al abogado de Málaga que le tramitó los papeles por diez mil pesetas y no se las cobró hasta que tuvo trabajo. Norma habló con mucho agradecimiento de su jefe, así como Igor hablaba de unos vecinos leridanos que les habían acogido como si fuera su familia. Han habido más comentarios como estos que señalan para nosotros este valor positivo y de buena fortuna.

En líneas generales, las personas de la muestra gozan de unas buenas estrategias relacionadas con los factores de ocupabilidad y empleabilidad, siguiendo el esquema que de estas estrategias hace Soledad Romero (2004).

\section{Preparación del proyecto migratorio}

\section{Planificación}

La inmensa mayoría de los inmigrantes procedentes de países extra-comunitarios disponen de una amplia información previa de España y sus posibilidades en cuanto al logro del proyecto migratorio, ya sea como destino o como tránsito a otros países de la UE o del ámbito de la OCDE (EE.UU., Canadá, etc.). Todo y así no podemos decir que dicha información sea fidedigna. La mayoría la aportan los medios de comunicación, la cinematografía, y 
a menudo la visión de los propios inmigrantes ${ }^{1}$ que dan una visión sesgada de una sociedad de consumo rica y abierta.

- En algunos casos los inmigrantes optan por un destino concreto tras un periodo largo de conocimiento y maduración del posible país de destino. "Ahora estoy tramitando mi marcha a Canadá, es un país verdaderamente multicultural, en donde nadie tiene problemas por hacer el ramadán, ser negro o lo que sea. El nivel profesional y cultural de la población es alto y su actitud hacia la inmigración es totalmente abierta y receptiva. Además allí tendré posibilidades profesionales reales" (Toufik, Argelia).

- En otros casos el destino está determinado por tener familiares de primer grado "Primero vino mi esposa" (Igor, Ucrania); segundo grado "Aquí estaban mis primas y tías" (Patricia, Perú); vecinos; "Aquí estaban varios chicos de mi pueblo" (Mohamed, Marruecos); o conocidos en el país "Un chico que conocí en Roma me habló de España” (Victor, Camerún).

- Finalmente podemos encontrar casos en los que la persona ni está ni en la primera ni en la segunda situación. Simplemente se ha planteado emigrar y decide el destino en función de aspectos coyunturales. "Viene aquí sólo, sin conocer a nadie, sin saber español, cogí el avión y pensé que ya me arreglaría” (Sergio, Casino).

\section{Canales y vías de regularización}

La actual normativa del Contingente requiere que la selección y contratación se realice en los países de origen.

En nuestro trabajo hemos conocido algún caso que en esta situación, y ante estas incógnitas han preferido pasar a la situación irregular hasta disponer de mayores garantías de retorno. "Ahora me gustaría encontrar trabajo, pero para obtener el permiso tengo que volver a Argelia y no me fío que me den el visado para volver a entrar... A un amigo mío ya le ha pasado esto y no me fio, así que de momento me quedaré aquí, buscaré trabajo en lo que pueda sin papeles y ya veremos lo que hago..." (Toufik. Argelia).

La contratación en país de origen, por sí misma, no es garante de integración sociolaboral. Aquellas personas depositarias de los ahorros para el pago de "retribuciones" y de las expectativas de toda su familia (extensa) en el país de origen, hipotecan muy a menudo todo su tiempo, dinero y esfuerzos en atesorar ingresos económicos que enviar a su familia. Cumpliendo un deber importantísimo en este tipo de culturas y logrando, a cambio, el reconocimiento más valioso que estas personas pueden atesorar. Otra atención especial merece el caso de los empleados contratados por contingente temporal. Ya que el propio contrato temporal limita las posibilidades y expectativas de integración.

Pero merece prestar atención al comentario que un representante de la Unió de Pagesos nos comentó al respecto de la integración de los trabajadores temporales "A nosotros, por diversos motivos, nos interesa que estos trabajadores se empadronen en los municipios mientras estén aquí, ... les ponemos viviendas confortables para que se sientan bien. Con

1. Esta acotación pretende aclarar que los inmigrantes que pueden aportar esta visión son aquellos que establecidos de manera regular o irregular, retornan periódicamente a sus lugares de origen cargados de presentes y mostrando una imagen autorrealizada ante sus coetáneos. 
ello conseguimos dos cosas, que no anden por la calle sucios y desarreglados (porque no tienen donde ducharse o lavar la ropa) o generando conflictos (porque no tienen donde estar). También hemos visto que si tienen una buena casa compran más en las tiendas del pueblo (sobre todo de alimentación). La verdad es que la mayoría de las tiendas que están cerca de estas viviendas y estos módulos han incrementado mucho el negocio. Los temporeros compran para cocinar, para limpiar... y eso le gusta a la gente de las tiendas" (Ramón, Unió de Pagesos).

\section{Proceso de exploración e inserción}

La persona que llega a nuestro país para encontrar trabajo se encuentra ante dos situaciones:

Acceso a la oferta de trabajo en su propio país

El inmigrante puede acceder a una oferta de trabajo en su propio país, mediante una agencia gubernamental, una intermediadora más o menos legal, etc. Desgraciadamente, las experiencias de selección en país de origen no han sido totalmente satisfactorias. "Allá sólo consigues algo si tienes una palanca 'enchufe', ninguna otra cosa vale" (Yaimy, Colombia). O la capacidad económica del candidato a emigrar que esté dispuesto a "avalar" su candidatura con algún "regalo". "Tuve que pagar para que me asignaran a la oferta", (Igor, Ucrania).

\section{Acceso a la oferta de trabajo en España}

La inmensa mayoría de las personas extranjeras que hemos entrevistado estaban ocupando puestos de trabajo de baja cualificación: Victor Palma y Victor Beteu trabajan de operario almacén, Igor de operario de mantenimiento, Fernando trabaja como operario textil, Aziz es soldador. Y todos ellos cuentan con unos estudios superiores a los secundarios, muchos están titulados otros no, pero en todos los casos han estudiado en la Universidad de su país. Y muchos han ocupado puestos cualificados y de alta responsabilidad en su país. No cabe duda que cuando hemos hablado con ellos su situación ocupacional no les satisfacía. Recordaban su nivel de cualificación y ahora se veían en una situación peor.

Respecto a la esperanza de llegar a trabajar en aquello de lo que estaban cualificados en sus países de origen hemos visto una actitud bastante realista en cuanto a las dificultades de lograrlo. "Era analista informático en Perú. Ahora soy mozo de almacén aquí. Soy mayor (44 años) para trabajar en esto mucho tiempo porque mi cuerpo no lo aguantará. Pero no me planteo llegar a trabajar de analista aquí. Soy mayor para empezar de cero y no aspiro a que me contraten directamente como analista" (Victor, Perú).

Lo que sí se plantean prácticamente todos ellos (hombres y mujeres, cualificados o no) es llegar a trabajar en una ocupación que les aporte mayor estabilidad y seguridad. Y si es posible, para algunos de ellos (los más jóvenes y cualificados), la posibilidad de realizar funciones más cualificadas. 
Estrategias de búsqueda de empleo

Nos atrevemos a afirmar que cuando el inmigrante llega a la ciudad de destino, lo primero que intenta localizar es una red de apoyo.

\section{Itinerarios de interés}

Creemos que los dos itinerarios de inserción que hemos podido identificar en este trabajo están más vinculados a factores de personalidad individual ${ }^{2}$ que a factores estrictamente ocupacionales o culturales.

\section{Itinerario social}

La red de acogida formal o informal le proporciona trabajo ${ }^{3}$, información sobre servicios de intermediación laboral (oficinas de trabajo, servicios de ocupación municipales, servicios de empleo de ONG y fundaciones, ETT, etc.) y una tutorización personal. "Mis compañeros de piso me hablaron de la ETT como una buena vía para encontrar un trabajo en fábrica. Así que fui, me apunté y al poco me llamaron para trabajar aqui" (Norma, Ecuador). Evidentemente, en estos casos, cuanto más integrado social y laboralmente estén los miembros de la red de acogida informal mayores expectativas de integración tendrá el inmigrante.

\section{Itinerario individual}

El segundo itinerario el inmigrante inicia su proceso de inserción individualmente. Él es el que busca información, localiza recursos y en definitiva gestiona y dirige individualmente su proceso de inserción.

No podemos ignorar que las personas que han utilizado este itinerario pertenecen a culturas eminentemente individualistas. "Cuando llegué a España, como que no existía el trabajo del contrato, me puse a buscar trabajo. Miré los anuncios de prensa pero no había casi nada en Lérida. Me fui al ayuntamiento a preguntar dónde encontrar trabajo y me dieron la dirección de la ETT, alli me hicieron una entrevista y prácticamente al día siguiente me llamaron para trabajar aqui" Igor, Ucrania.

Queremos finalizar, por el interés que conlleva, acercarnos al concepto de integración desde los propios inmigrantes.

2. Recordemos a autores como Triandis (1985) y Brislin (1994) que diferenciaban entre personalidades ideocéntricas (orientadas al logro, a la iniciativa individual y a la exploración) y personalidades allocéntricas (orientadas a la relación, al proceso, a la conservación).

3. Hemos visto mucho esto en la inserción de la mujer latinoamiericana. Es su red de acogida quien la pone en contacto con las empresas y le llega a negociar los empleos, no es que pacten el salario u otras condiciones, nos referimos que le buscan el trabajo. El caso del colectivo paquistaní de Barcelona que está ocupando la actividad de reparto de butano a domicilio también es significativo. 


\section{Concepto de integración de la población estudiada}

Nosotros hemos considerado que un trabajador inmigrante estará integrado cuando tenga una relación laboral regular y con una cierta duración temporal, desarrolle adecuadamente sus funciones, mantenga unas relaciones personales con sus compañeros que excedan, aunque sea mínimamente, el ámbito profesional y en definitiva está valorado positivamente por parte de la empresa.

Cuando le hemos preguntado a ellos por la integración han contestado varias cosas, tanto desde la perspectiva positiva "me encanta el trabajo que hago, me llevo bien con mis compañeras y se siento bien aquí. Por mi me quedaría para siempre en esta empresa" (Norma, Egarfil). "Para mi estar en esta empresa es la culminación de todos mis sueños, somos los mejores profesionales, todos los compañeros y los jefes somos una piña; damas y caballeros que compartimos la oportunidad de trabajar en la mejor firma del mundo" (Hernán, Hotel Arts).

Como desde la negativa "mi jefe era muy buena persona, el trabajo me gustaba pero llegó un momento en que no pude aguantar más los insultos y maltrato de mis compañeros, no podía más y aunque ganaba dinero y podría haber estado alli para siempre me marché" (Aziz, Marrueco).

Pero quizás la definición de integración que más nos ha gustado ha sido la que nos dio Aziz "Cuando no hay relación de verdad con los españoles todo es muy bueno o todo es muy malo. Yo que me considero integrado, trabajo, tengo mi novia española y otros amigos españoles también, los conozco lo suficiente como para haber visto lo bueno, y también lo malo. Porque cuando tienes una relación de verdad al igual que tienes buenos momentos, también los tienes malos".

\section{Conclusiones}

Como punto final del presente trabajo vamos a presentar las siguientes conclusiones acerca de nuestra perspectiva sobre la integración socio-laboral del inmigrante en España:

- Las diferencias en cuanto a las estrategias de integración y sus logros están más determinadas por los niveles de cualificación en el país de origen que por las propias diferencias culturales.

- Dada la situación actual del mercado de trabajo, tanto regular como irregular, ni la formación ni el origen cultural parecen elementos clave para el acceso a una ocupación.

- La cualificación de base y las competencias para la empleabilidad se muestran como requisitos fundamentales para acceder a un empleo con perspectivas de integración y desarrollo profesional.

- Se han detectado mayores conflictos de aceptación y rechazo entre los compañeros del inmigrante que entre los propios responsables y mandos. Es este aspecto, la dirección emerge de nuevo como factor clave.

- El presente estudio puede ayudar a los orientadores que quieran incorporar una orientación multicultural (Castellano Pérez, 2005) en su realización profesional gracias a una comprensión más profunda de los procesos de inserción de la población inmigrante. 


\section{Referencias bibliográficas}

Ahmed, A. S. (2002). Migrations et développement en Méditerranée: Leçons et perspectives à la lumière de l'accord de Barcelone. Simposi Internacional Europa - mediterrània. Polítiques d'Immigració, Barcelona, Institut Català de la Mediterrània. Universitat Pompeu Fabra.

Aja, E. (1994). Migrantes, refugiados y cooperación internacional. Una aportación para la Conferencia Internacional sobre la Población y el Desarrollo. CIPD, Ginebra.

Aneas, A. y Perez, N. (1999). Conseguir un empleo: ¿Índice de calidad en la Formación Ocupacional? Comunicación en el Congreso Internacional de Calidad educativa. Empleo e interculturalismo en el Camino de Santiago. Santiago de Compostela.

Aneas, A. (2003). Competencias interculturales transversales en la empresa: un modelo para la detección de necesidades formativas. Tesis doctoral. Universidad de Barcelona.

Arango, J. (2002). El Mediterráneo, entre la mundialización y la diversidad ideosincrática. Simposi Internacional Europa - mediterrània. Polítiques d'Immigració. Barcelona, Institut Català de la Mediterrània. Universitat Pompeu Fabra.

Baldwin-Edwards, M. (2002). Inmigration and European Welfare systems: A frame work for comparative analysis. Simposi Internacional Europa - mediterrània. Polítiques d'Immigració, Barcelona, Institut Català de la Mediterrània. Universitat Pompeu Fabra.

Blanch, J. M. (1990). Del viejo al nuevo paro. Un análisis psicológico y social. Barcelona, PPU.

Böcker, A. y. H., T (1998). Asylum, Mogration to the European Unio: Patterns of origin and destination. Luxemburgo, Oficina para las publicaciones oficiales de las Comunidades Europeas.

Castellano Pérez, J.L. (2005). "Competencias interculturales en Servicios de Orientación para el desarrollo de la carrera”. Revista Española de Orientación y Psicopedagogía. Vol. 16, $\mathrm{n}^{\mathrm{o}} 1^{\circ}$ semestre, 13-29.

Castells, M. (2000). La era de la información. Madrid, Alianza Editorial.

CERD Comité para la Eliminación de la Discriminación Racial (1998). Decimoquinto informe periódico que los Estados Partes debían presentar en 1998: Spain, Naciones Unidas.

Comisión de Ayuda al Refugiado (2000). Análisis del Proyecto de Ley de Reforma de la Ley Orgánica 4/2000, del 11 de enero, sobre los derechos y libertades de los extranjeros en España y su integración social.

Comisión Europea (2000). Carta de los Derechos Fundamentales de la unión Europea. Biarritz, Unión Europea.

Daugareilh, I. (2000). Los derechos fundamentales de los trabajadores de la Unión Europea. Université Montesquieu Boredeux IV.

Donoso y Aneas (2004). "Multiculturalitat a l'empresa. Emergencia de la formació Intercultural". Temps d'educación, 27: 105-117.

Donoso, T. y Figuera, P. (2007). "Niveles de diagnóstico en los procesos de inserción y orientación profesional". Revista electrónica de investigación psicoeducativa, http://www.investigacion-psicopedagogica.org/revista/new/index.php volumen 5, (1) no 11.

Figuera, Massot y Aneas (1989). El desarrollo Personal. Iniciativa Comunitaria Youthstart. Ayuntamiento de Barcelona: Área de Juventud.

Figuera, P. (1996). La inserción del universitario en el mercado de trabajo. Barcelona, EUB.

Garcia, M. y Figuera, P. (2000). "La Garantia Social, un recurs de lluita contra la exclusió social". Temps d'Educació, 24: 241-262.

Hofstede, G. (1999). Culturas y organizaciones. Madrid, Alianza Editorial. 
Ioé, C. (2000). "La immigració estrangera a Espanya, 2000”. La immigració estrangera a Espanya. Els reptes educatius. F. 1. Caixa". Barcelona, Fundació "la Caixa".

Jurado, P. (1990) La integración socio-laboral de los sujetos con retraso mental ligero y de capacidad intelectual límite en el ámbito de la educación especial. Tesis Doctoral Inédita. Universidad Autónoma de Barcelona.

Rísquez, A. (2004). "The effects of cross-cultural differences on career development and vocational counseling: An empirical study of the factors influencing motivation of Irish and Spanish workers". Revista Española de Orientación y Psicopedagogía. Vol. 15, nº 1, 1 semestre: 11-31.

Romero, S. (2004). “Aprender a construir proyectos profesionales y vitales". Revista Española de Orientación y Psicopedagogía.Vol. 15, nº 2, 2 semestre: 337-354.

Triandis, H. C., Leung, K., Villareal, M. y Clark, F. L. (1985). "Allocentric vs. ideocentric tendencies: convergent and discriminant validation". Journal of Research in Personality, 19: 395-415.

(UNHCR) (2001). International Migration, Racism, Discrimination and Xenophobia A. S. (2002).

Fecha de recepción:26-06-06

Fecha de revisión: 20-07-07

Fecha de aceptación: 27-02-08 